\title{
Erratum to: A Profile of Chinese Family Therapists
}

\author{
Yee Tak Sze $\cdot$ Juan Hou $\cdot$ Xiaoyi Fang
}

Published online: 6 May 2014

(C) Springer Science+Business Media New York 2014

\section{Erratum to: Contemp Fam Ther}

DOI 10.1007/s10591-014-9299-1

In the original version of this article, an article note was unfortunately not submitted and published.

The note should read as:

Yee Tak Sze and Juan Hou are first authors.

The online version of the original article can be found under doi:10.1007/s10591-014-9299-1.

Y. T. Sze $\cdot$ X. Fang $(\bowtie)$

Institute of Developmental Psychology, Beijing Normal

University, Beijing, People's Republic of China

e-mail: fangxy@bnu.edu.cn

Y. T. Sze

e-mail: sze.maranda@aliyun.com

J. Hou

Department of Philosophy, Anhui University, Hefei, Anhui,

People's Republic of China

e-mail: daisyhoujuan@gmail.com 